\title{
ESTUDO SOBRE A EVOLUÇÃO DO RISCO DE INFECÇÃO TUBERCULOSA EM ÁREA COM ELEVADA COBERTURA POR BCG
}

Gilberto Ribeiro Arantes * Stella Maria Costa Nardy * Jannete Nassar **

ARANTES, G.R. et al. Estudo sobre a evolução do risco de infecção tuberculosa em área com elevada cobertura por BCG. Rev. Saúde públ., S. Paulo, $19: 95-107,1985$.

RESUMO: A partir da prevalência de infecção tuberculosa em escolares com 7 anos de idade, calculou-se a taxa de redução do risco anual de infecção na cidade de São Paulo (Brasil), entre 1974 e 1982. Nesse período o declínio médio foi de $5 \%$ ao ano. Nas 59 escolas municipais pesquisadas não houve correlação entre a cobertura de vacinação BCG e a prevalência de infecção natural em não-vacinados, à idade estudada. A alergia tuberculínica no grupo de crianças vacinadas, que recebeu a vacina em alguma idade anterior entre o 19 e o $6^{\circ}$ ano de vida, revelou-se 2,5 vezes mais intensa do que a alergia no grupo de mesma idade ( 7 anos), não vacinado previamente. Foram feitos comentários quanto à impropriedade do material utilizado com vistas ao cálculo do verdadeiro valor do risco de infecção tuberculosa na área em questão.

UNITERMOS: Tuberculose, risco de infecção. Vacina BCG. Teste tuberculínico. Hipersensibilidade tardia.

\section{INTRODUÇĀO}

O risco anual de infecção tuberculosa guarda estreita relação com a incidência anual de casos bacilíferos, tendo sido demonstrada correspondência matemática entre ambos $24, * * *$.

E um indicador que exprime a força de ataque da tuberculose na comunidade, bastante sensível às suas variações, e por isso considerado o melhor indicador isolado para aferir a intensidade do problema, acompanhar sua tendência e avaliar o efeito global das ações exercidas para o controle ${ }^{4,23}$. Embora possa ser calculado diretamente em estudos longitudinais, a maneira mais fácil e menos onerosa de fazê-lo é a indireta, a partir da prevalência de infecção ${ }^{16}$. Como se sabe, a proporção de pessoas infectadas, em uma determinada idade, é expressão dos riscos de infecção aos quais as mesmas foram submetidas desde o nascimento; por esse motivo, o risco calculado nessa idade representa o risco médio nos anos anteriores ${ }^{25}$. Para se obter o risco atual determina-se a tendência do mesmo nos anos precedentes e, a partir do dado médio, extrapola-se-o para o ano em curso.

A tendência no tempo pode ser estimada com base na prevalência de infecção em duas ou mais idades num mesmo ano, ou numa só idade em anos diferentes ${ }^{16,25}$.

\footnotetext{
- Do Departamento de Epidemiología da Faculdade de Saúde Pública da Universidade de São Paulo - Av. Dr. Arnaldo, 715 - 01255 - São Paulo, SP - Brasil.

* Da Secretaria de Estado da Saúde - Av. Dr. Arnaldo, 351 - 01246 - São Paulo, SP - Brasil.

** K. Styblo - The relationship between the annual risk of tuberculous infection and the incidence of smear-positive pulmonary tuberculosis. Tuberculosis Surveillance Research Unit Progress Report, 1982. [não publicado]
} 
ARANTES, G. R, et. al. Estudo sobre a evolução do risco de infecção tuberculosa em área com elevada cobertura por BCG. Rev. Saúde públ., S. Paulo, 19:95-107, 1985.

Uma vez que o risco pode variar com o tempo, o risco médio deve abranger um período de vida curto, daí a preferência pelo início da idade escolar, ou pouco mais, dependendo da realidade epidemiológica; além do que, nessa faixa etária, as variações com a idade são menores ${ }^{16}$ e por isso, para fins práticos, podem ser ignoradas.

No Estado de São Paulo, cujo contingente de doentes representa quase a quarta parte do estoque do país, as pesquisas a respeito são pouco numerosas, tendo sido algumas delas realizadas em populações de demanda, impróprias para esse fim $1,2,5,8,13,19$. Os levantamentos tuberculínicos realizados em escolares do município de São Paulo, de 1970 a 1976, nos estudos que precederam a implantação da vacina BCG pela via intradérmi$\mathrm{ca}^{7,8}$, constituem material que, devidamente tratado, permite alguns cálculos do risco de infecção relativo àquela época; e a repetição de pelo menos um desses inquéritos, no presente, em condições comparáveis, possibilitaria o cálculo da tendência média desde então.

Com a implantação da vacinação intradérmica, capaz de produzir uma alergia tuberculínica intensa e duradoura, apenas os escolares ainda não vacinados seriam utilizáveis para esse fim. Existem dúvidas quanto à validade de se calcular o risco de infecção, em não vacinados, quando a cobertura de vacinação BCG é elevada ${ }^{16}$ pois os mesmos podem representar um grupo sócio-econômico diferente, mais sujeito aos fatores de risco. Todavia, se ao atingirem a idade escolar, os vacinados nos primeiros anos de vida apresentarem alergia idêntica a dos não vacinados, talvez eles possam ser também utilizados, superando-se com isso os inconvenientes de uma possível seleção.

Além disso, é possível que a presença de uma substancial parcela de população vacinada, em uma comunidade, possa afetar a incidência e prevalência de infecção entre os não vacinados da mesma comunidade ${ }^{9}$.

Visando a contribuir para o esclarecimento de algumas dessas questões e considerando a necessidade de se conhecer melhor a evolução da endemia, pelo menos no município de São Paulo, planejou-se esta pesquisa, cujos objetivos foram os seguintes:

1. Determinar a tendência do risco de infecção em crianças com 7 anos de idade, não vacinadas, matriculadas em escolas primárias, no município de São Paulo.

2. Comparar a alergia tuberculínica dos vacinados com a dos não vacinados, à idade escolar.

3. Verificar se nas escolas examinadas há correlação entre a cobertura vacinal e a prevalência de infecção tuberculosa nos não vacinados.

\section{MATERIAL e METODOS}

A capital do Estado de São Paulo é o município de mesmo nome, localizado no planalto paulista. Estendendo-se por $1.520 \mathrm{~km}^{2}$, compreende cerca de 56 unidades administrativas (distritos ou subdistritos). Sua população era de 3.709.274 habitantes em 1960, 5.978.977 em 1970 e 8.493 .598 em 1980, distribuída em 3 zonas - "central", "intermediária" e "periférica" - de acordo com a sua posição geográfica e segundo as taxas de crescimento populacional, grau de urbanização, condições de saneamento básico e nível sócio-econômico ${ }^{12}$. O cresccimento populacional, muito grande nas zonas intermediária e periférica, foi devido, em grande parte, ao afluxo de migrantes, na sua maioria pessoas de nível educacional e econômico reduzido ${ }^{12}$.

A rede pública de ensino primário era constituída por 486 escolas estaduais e 218 municipais no começo da década de 70; em 1982 essas redes atingiram respectivamente 715 e 278 unidades. Aproximadamente $70 \%$ das escolas munici- 
ARANTES, G. R. et. al. Estudo sobre a evolução do risco de infecção tuberculosa em área com elevada cobertura por BCG. Rev. Saúde públ., S. Paulo, 19:95-107, 1985.

pais e $50 \%$ das estaduais se localizam na "periferia", isto é, naquelas zonas onde predominam precárias condições sócioeconômicas e sanitárias 11,20 .

População de estudo - Constitui-se de alunos de 59 escolas municipais de
$1 .^{\circ}$ grau, a maioria delas (39) localizadas nas zonas central e intermediária e algumas (20) na zona periférica; como se pode notar na Fig. 1, a maior parte da zona periférica, isto é, as áreas a noroeste, leste e sul, não esteve representada.

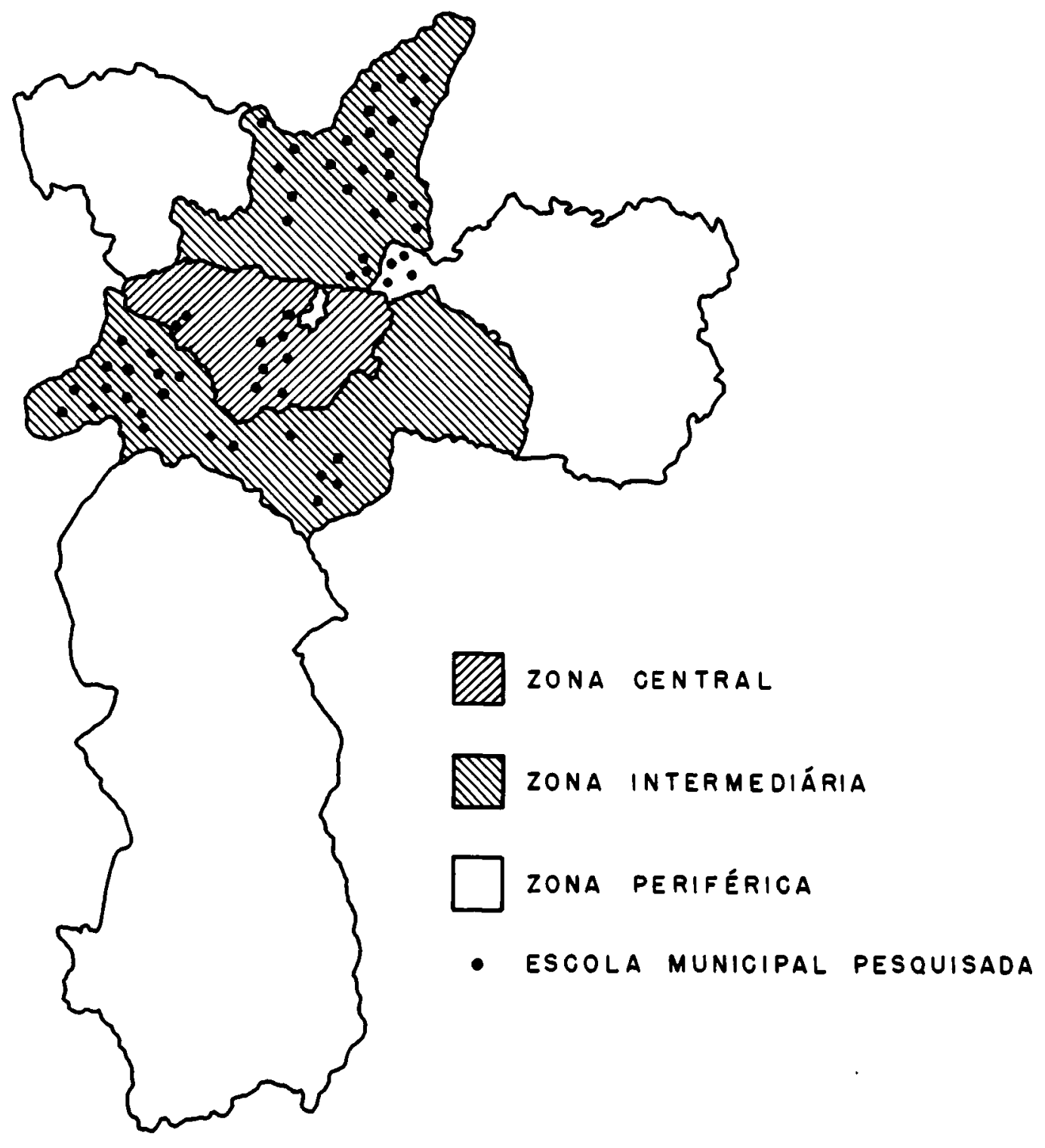

Fig. 1 - Zonésmento da cidade de São Paulo, segundo a posição geográfica, urbanização, condiçōes de saneamento básico e distribuiçăo da renda.

Fonte: Leser e Barbosal2 e Monteiro e col. ${ }^{14}$ 
ARANTES, G. R. et. al. Estudo sobre a evolução do risco de infecção tuberculosa em área com elevada cobertura por BCG. Rev. Saúde públ., S. Paulo, 19:95-107, 1985.

Essas mesmas escolas fizeram parte de levantamento tuberculínico realizado no ano de 1974. Foram incluídos apenas os alunos com 7 anos completados no início das aulas $(02 / \mathrm{Fev} / 82)$, cursando a primeira série do primeiro grau durante o primeiro semestre letivo de 1982, com autorização paterna para fazer parte da pesquisa. Ao serem inscritos, era solicitado aos alunos que apresentassem a "caderneta de vacinação", embora esta condição não fosse eliminatória. Os dados de identificação, data de vacinação BCG ou ausência de anotação à respeito, bem como a informação sobre a apresentação ou não da Caderneta eram anotados em fichas individuais. A equipe técnica de campo foi constituída por enfermeiras e auxiliares de saúde pública, padronizadas e aferidas, e educadoras algumas delas participantes da equipe de 1974; a suprevisão foi feita por um dos autores do presente trabalho, nas duas oportunidades.

Após a pesquisa de cicatriz vacinal em ambos os braços, anotando-se na ficha a presença ou ausência da mesma, aplicou-se o teste tuberculínico segundo normas técnicas em vigor ${ }^{17}$. A leitura era feita $72 \mathrm{~h}$ depois, com anotação do resultado em milímetros. A apuração dos dados foi realizada manualmente.

"A proporção de uma coorte que ainda permanece não infectada numa certa idade é igual ao produto dos riscos anuais de escapar à infecção experimentados pela coorte até aquela idade" ${ }^{25}$, o que se pode expressar matematicamente por ${ }^{28}$ :

$$
\begin{aligned}
& \mathrm{N}_{\mathrm{n}}=\mathrm{N}_{0} \times(1-\mathrm{r})^{\mathrm{n}} \\
& \mathrm{n}=\text { idade da coorte em questão, } \\
& \mathrm{N}_{\mathrm{n}}=\begin{array}{l}
\text { proporção de não infectados } \\
\text { à idade atual, }
\end{array} \\
& \mathrm{N}_{\mathrm{o}}=\begin{array}{l}
\text { proporção de não infectados à } \\
\text { à idade inicial, }
\end{array}
\end{aligned}
$$$$
\text { onde, }
$$

$$
r=\begin{aligned}
& r i s c o \\
& \text { entre "o" e " } \mathrm{n} \text { ". }
\end{aligned}
$$

Para o cálculo do risco de infecção derivou-se da equação supra a seguinte expressão:

$$
r=1-N_{n}^{1 / n}
$$

Admitindo-se que o declínio do risco de infecção tenha ocorrido segundo uma curva exponencial, como se observou em outras áreas ${ }^{26}$, a redução $(R)$ no período compreendido entre os dois anos estudados foi calculada pela expressão:

$$
\mathrm{R}=1-(\mathrm{rf} / \mathrm{ri})^{1 / \mathrm{t}}
$$

onde,

$$
\begin{aligned}
& \mathrm{rf}= \text { risco de infecção no final do } \\
& \text { período }
\end{aligned}
$$

\section{RESULTADOS}

$\mathrm{Na}$ Tabela 1 constam dados relativos a levantamentos tuberculínicos realizados de 1970 a 1976 em escolas públicas no município de São Paulo.

$\mathrm{Na}$ Tabela 2 pode-se comparar a prevalência de reatores fortes aos 7 anos de idade, entre alunos da primeira série do primeiro grau, de escolas municipais, segundo o semestre letivo do ano de 1974. $\mathrm{Na}$ Fig. 2 é apresentada a distribuição da população de estudo em 1982, segundo a presença de cicatriz vacinal, apresentação da caderneta e antecedente vacinal. Naquele ano havia 5.827 alunos com 7 anos completos, na primeira série das 59 escolas estudadas, no primeiro semestre letivo; o teste tuberculínico foi aplicado em 5.249 (90\%) e lido em $4.914(94 \%)$. 
ARAN'TES, $G$. R. et. al. Estudo sobre a evolução do risco de infecção tuberculosa em área com elevada cobertura por BCG. Rev. Saúde públ., S. Paulo, 19:95-107, 1985.

\section{TABELA 1}

Distrbiuição percentual dos reatores fortes $(10 \mathrm{~mm} e+)$ ao teste tuberculínico padronizado por idade e ano do levantamento, alunos do primeiro ano primário, Escolas Públicas no município de São Paulo, 1970-1976.

\begin{tabular}{cccccccc}
\hline \multicolumn{7}{c}{ Ano do Levantamento } \\
\cline { 2 - 8 } Idade & $\begin{array}{c}1970 \\
\%\end{array}$ & $\begin{array}{c}1971 \\
\%\end{array}$ & $\begin{array}{c}1972 \\
\%\end{array}$ & $\begin{array}{c}1973 \\
\%\end{array}$ & $\begin{array}{c}1974 \\
\%\end{array}$ & $\begin{array}{c}1975 \\
\%\end{array}$ & $\begin{array}{c}1976 \\
\%\end{array}$ \\
\hline anos) & 5,0 & 4,0 & 4,3 & 9,4 & 5,3 & 5,3 & 13,5 \\
6 & 5,6 & 5,1 & 5,5 & 5,9 & 6,3 & 7,5 & 7,3 \\
7 & 8,8 & 6,7 & 9,0 & 7,8 & 7,8 & 5,8 & 7,7 \\
8 & 8,9 & 8,8 & 12,1 & 11,7 & 10,9 & 12,4 & 10,0 \\
9
\end{tabular}

Fonte: Arquivo de Tuberculose do Departamento de Epidemiologia da Faculdade de Saúde Pública da USP.

TABELA 2

Prevalência de reatores fortes $(10 \mathrm{~mm}$ e + ) ao teste tuberculínico padronizado, por semestre letivo, alunos do primeiro ano primário com 7 anos de idade. Escolas municipais, São Paulo (SP), 1974.

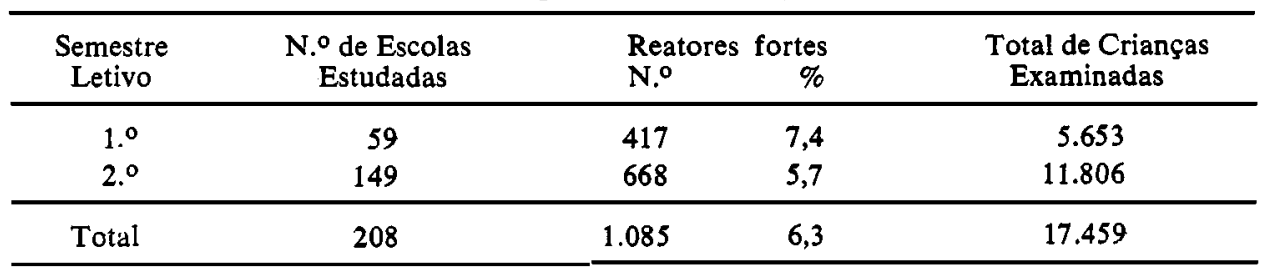

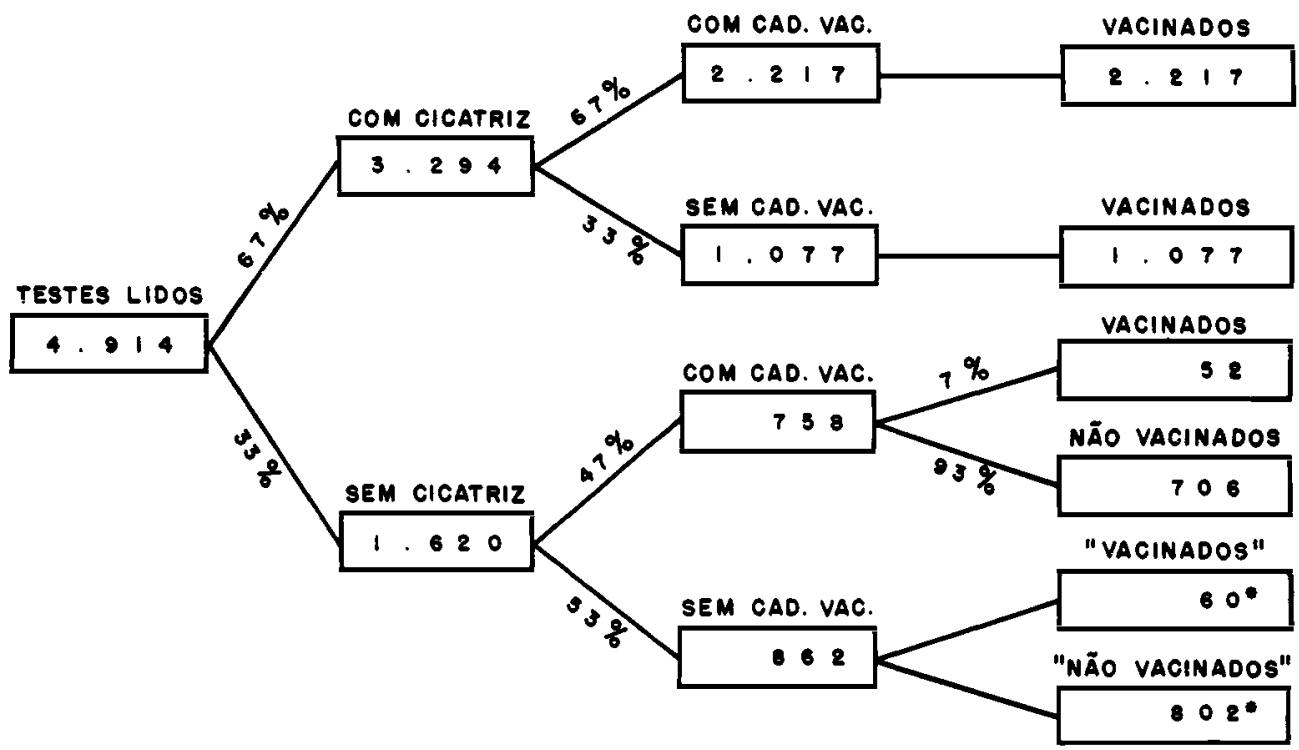

Fig. 2 - Distribuição dos escolares que completaram o teste tuberculínico, segundo a preserı̧̧a de cicatriz vacinal, apresentação da caderneta de vacinação e antecedente vacinal.

- Dados estimados admitindo-se que a distribuição tenha sido a mesma observada nos 758 com caderneta de vacinação; para a confecçāo das Tabelas e dos perfis tuberculícos foram considerados como não vacinados. 
ARANTES, G. R. et. al. Estudo sobre a evolução do risco de infecção tuberculosa em área com elevada cobertura por BCG. Rev. Saúde públ., S. Paulo, 19:95-107, 1985.

Destes, 3.294 (67\%) apresentavam cicatriz vacinal atribuível ao BCG, e por isso classificados como vacinados, inclusive os 1.077 que não apresentaram a respectiva caderneta. Os 1.620 sem cicatriz, descontados 52 com o dado positivo na caderneta, foram considerados não vacinados e os respectivos resultados do teste tuberculínico foram utilizados para o cálculo do risco de infecção.

A prevalência de reatores fortes ao teste tuberculínico, por Região Municipal de Ensino, é apresentada na Tabela 3; nas Tabelas 4 e 5 esses mesmos dados são apresentados segundo o Distrito/Subdistrito de Paz do município.

TABELA 3

Prevalência de reatores fortes $(10 \mathrm{~mm} \mathrm{e}+)$ ao teste tuberculínico padronizado, por Regional Municipal de Ensino, alunos do primeiro ano primário com 7 anos de idade, nz̃o vacinados. por ano do levantamento -1974 e 1982, São Paulo (SP).

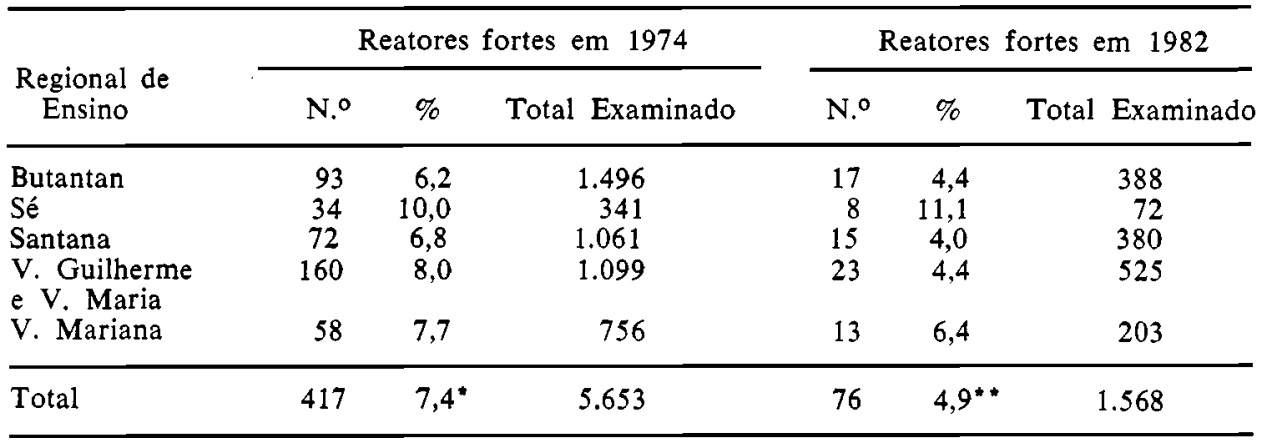

- Risco de infecção $=1,09 \%$

* Risco de infeç̧ão $=0,71 \%$

TABELA 4

Prevalência de Reatores fortes ao teste tuberculínico padronizado nos Distritos/Sub-distritos de $\mathrm{Paz}$ onde apenas uma escola foi estudada. (Alunos do primeiro ano primário, com 7 anos de idade, não vacinados com BCG). Município de São Paulo, 1974 e 1982.

\begin{tabular}{|c|c|c|c|c|c|}
\hline \multirow{2}{*}{$\begin{array}{l}\text { Distrito ou } \\
\text { Sub-distrito }\end{array}$} & \multirow{2}{*}{$\begin{array}{c}\text { N. }{ }^{\circ} \text { de Escolas } \\
\text { Estudadas }\end{array}$} & \multicolumn{2}{|c|}{1974} & \multicolumn{2}{|c|}{1982} \\
\hline & & $\begin{array}{c}\text { N. }{ }^{\circ} \text { de Alunos } \\
\text { Examinados }\end{array}$ & $\begin{array}{l}\% \text { de Reato- } \\
\text { res fortes }\end{array}$ & $\begin{array}{l}\text { N. }{ }^{\circ} \text { de Alunos } \\
\text { Examinados }\end{array}$ & $\begin{array}{l}\% \text { de Reato- } \\
\text { res fortes }\end{array}$ \\
\hline $\begin{array}{l}\text { V. Mariana } \\
\text { Pari } \\
\text { Aclimação } \\
\text { Lapa } \\
\text { Saúde } \\
\text { V. Madalena } \\
\text { Indianópolis } \\
\text { Jardim Pta. } \\
\text { Bela Vista } \\
\text { Liberdade }\end{array}$ & $\begin{array}{l}1 \\
1 \\
1 \\
1 \\
1 \\
1 \\
1 \\
1 \\
1 \\
1\end{array}$ & $\begin{array}{c}28 \\
54 \\
65 \\
68 \\
69 \\
79 \\
87 \\
94 \\
101 \\
121\end{array}$ & $\begin{array}{r}3,6 \\
7,4 \\
4,7 \\
5,8 \\
2,9 \\
6,0 \\
10,4 \\
5,3 \\
18,8 \\
6,6\end{array}$ & $\begin{array}{r}9 \\
13 \\
13 \\
9 \\
13 \\
8 \\
17 \\
25 \\
13 \\
33\end{array}$ & $\begin{array}{l}22,2 \\
15,4 \\
15,4 \\
\overline{15,4} \\
- \\
- \\
8,0 \\
7.7 \\
9.1\end{array}$ \\
\hline Total & 10 & 766 & 7,8 & 153 & 9,1 \\
\hline
\end{tabular}


ARANTES, G. R. et. al. Estudo sobre a evolução do risco de infecção tuberculosa em área com elevada cobertura por BCG. Rev. Saúde públ., S. Paulo, 19:95-107, 1985.

\section{TABELA 5}

Prevalência de reatores fortes ao teste tuberculínico padronizado nos Distritos/Sub-distritos de Paz onde mais de uma escola foi estudada. (Alunos do primeiro ano primário, com 7 anos de idade, não vacinados com BCG). Município de São Paulo, 1974 e 1982.

\begin{tabular}{|c|c|c|c|c|c|}
\hline \multirow{2}{*}{$\begin{array}{l}\text { Distrito ou } \\
\text { Sub-Distrito }\end{array}$} & \multirow{2}{*}{$\begin{array}{l}\text { N.o de Escolas } \\
\text { Estudadas }\end{array}$} & \multicolumn{2}{|c|}{1974} & \multicolumn{2}{|c|}{1982} \\
\hline & & $\begin{array}{c}\text { N.o de Alunos } \\
\text { Examinados }\end{array}$ & $\begin{array}{l}\% \text { de Reato- } \\
\text { res fortes }\end{array}$ & $\begin{array}{l}\text { N. }{ }^{\circ} \text { de Alunos } \\
\text { Examinados }\end{array}$ & $\begin{array}{l}\% \text { de Reato- } \\
\text { res fortes }\end{array}$ \\
\hline Ibirapuera & 2 & 181 & 9,4 & 54 & 5.5 \\
\hline V. Guilherme & 3 & 191 & 10,5 & 48 & 6,2 \\
\hline Jabaquara & 4 & 391 & 7,4 & 110 & 5,4 \\
\hline V. Maria & 4 & 405 & 9,6 & 101 & 7,9 \\
\hline Santana & 5 & 527 & 7,6 & 157 & 3,2 \\
\hline Butantan & 14 & 1.255 & 6,3 & 346 & 4,3 \\
\hline Tucuruvi & 17 & 1.937 & 6,9 & 599 & 3,7 \\
\hline Total & 49 & 4.887 & 7,3 & 1.415 & 4,4 \\
\hline
\end{tabular}

O risco anual de infecção aos 7 anos de idade, que era de $1,09 \%$ em 1974, caiu para $0,71 \%$ em 1982 , correspondendo essa diferença a um declínio médio anual de $5 \%$.

$\mathrm{Na}$ Tabela 6 são apresentados o diâ- metro médio e as percentagens de reaçōes entre 5 e $9 \mathrm{~mm}$ e com 10 e mais milímetros nos vacinados, segundo o tempo decorrido após a vacinação, e os respectivos totais, em comparação com os dados relativos aos não vacinados.

\section{TABELA 6}

Diâmetro médio, "reações fracas" e "reações fortes" ao teste tuberculínico padronizado, em alunos do primeiro ano primário com 7 anos de idade, vacinados com BCG I.D., segundo o tempo decorrido após a vacinação. Escolas municipais, São Paulo, SP, 1982.

\begin{tabular}{ccccc}
\hline $\begin{array}{c}\text { Tempo decorrido } \\
\text { após a vacinação } \\
\text { (anos) }\end{array}$ & $\begin{array}{c}\text { N. }{ }^{\circ} \text { de } \\
\text { Exami- } \\
\text { nados }\end{array}$ & $\begin{array}{c}\text { dm médio } \\
(\mathrm{mm})\end{array}$ & $\begin{array}{c}\% \text { reações } \\
\text { a } 9 \mathrm{~mm}\end{array}$ & $\begin{array}{c}\% \text { reações } \\
10 \mathrm{e}+\mathrm{mm}\end{array}$ \\
\hline 1 & 212 & 6,9 & 21,2 & 32,6 \\
2 & 396 & 5,7 & 22,0 & 21,4 \\
3 & 489 & 4,8 & 22,7 & 15,3 \\
4 & 675 & 5,0 & 20,0 & 17,9 \\
5 & 292 & 4,9 & 18,2 & 21,9 \\
6 & 87 & 5,6 & 19,5 & 23,5 \\
7 & 68 & 5,2 & 13,3 & 20,1 \\
\hline Total & 2.219 & 5,2 & 20,6 & 4,9 \\
\hline Não vacinados & 1.568 & 2,1 & 2,7 &
\end{tabular}

Os perfís tuberculínicos traçados com os resultados individuais obtidos no total de vacinados e não vacinados podem ser vistos nas Figs. 3 e 4, respectivamente.
Finalmente, a prevalência de crianças com cicatriz vacinal e a prevalência de infecção nos não vacinados, nas 59 escolas estudadas, são apresentadas de for- 
ARANTES, G. R. et. al. Estudo sobre a evolução do risco de infecção tuberculosa em área com elevada cobertura por BCG. Rev. Saúde públ., S. Paulo, 19:95-107, 1985.

ma agrupada na Tabela 7 e de forma coeficiente de correlação, " $r$ ", entre as individualizada no diagrama da Fig. 5. O duas variáveis, foi igual a 0,024 .

\section{TABELA 7}

Distribuição das escolas municipais estudadas, segundo a prevalência de cicatriz vacinal (BCG)

e a prevalência de infeç̧ão entre os alunos não vacinados, com 7 anos de idade, do primeiro ano. São Paulo, SP, 1982.

\begin{tabular}{lcccccr}
\hline \multirow{2}{*}{$\begin{array}{c}\text { Cobertura } \\
\text { BCG }(\%)\end{array}$} & \multicolumn{6}{c}{ Prevalência de Infecção em não vacinados $(\%)$} \\
\cline { 2 - 7 } & $0-4$ & $5-9$ & $10-14$ & $15-19$ & $20-24$ & Total \\
\hline $40-49$ & 1 & 1 & 1 & - & - & 3 \\
$50-59$ & 5 & 1 & - & - & - & 6 \\
$60-69$ & 14 & 8 & - & 1 & - & 23 \\
$70-79$ & 7 & 7 & 3 & 1 & 1 & 19 \\
$80-89$ & 5 & 2 & - & 1 & - & 8 \\
\hline Total & 32 & 19 & 4 & 3 & 1 & 59 \\
\hline
\end{tabular}

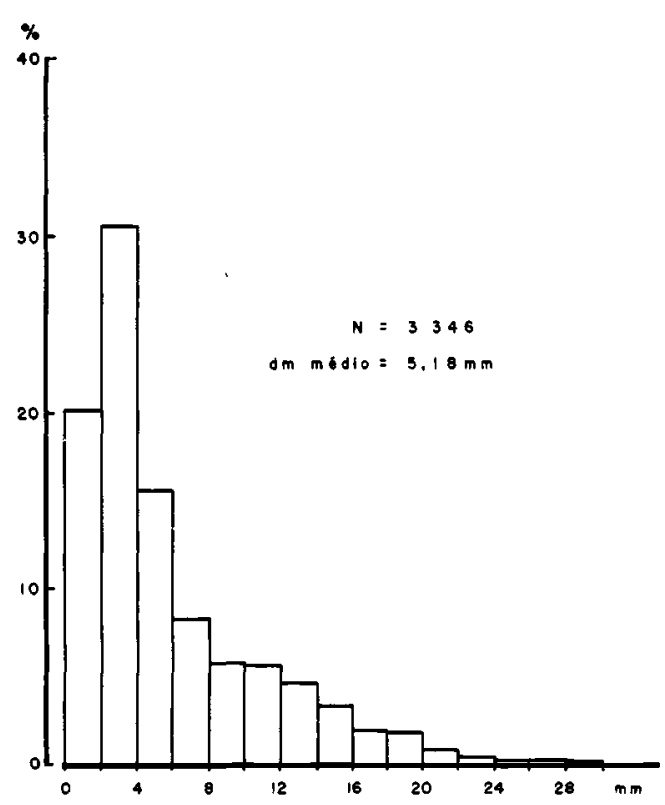

Fig. 3 - Distribuição percentual das reaçōes ao teste tuberculínico padronizado $(2 \mathrm{U}$. $T$. de PPD rt 23) em escolares com 7 anos de idade, vacinados anteriormente com BCG Id. São Paulo, 1982.

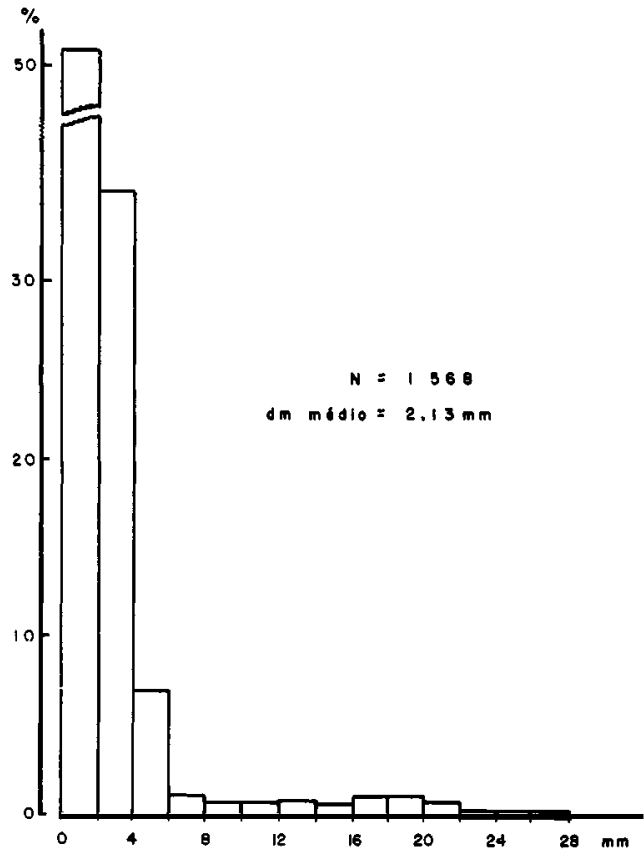

Fig. 4 - Distribuiçāo percentual das reaçōes ao teste tuberculínico padronizado (2 U.T. de PPD rt 23) em escolares com 7 anos de idade, nåo vacinados antericrmente cờn BCG id. São Paulo, 1982. 
ARANTES, G. R. et. al. Estudo sobre a evolução do risco de infecção tuberculosa em área com elevada cobertura por BCG. Rev. Saúde públ., S. Paulo, 19:95-107, 1985.

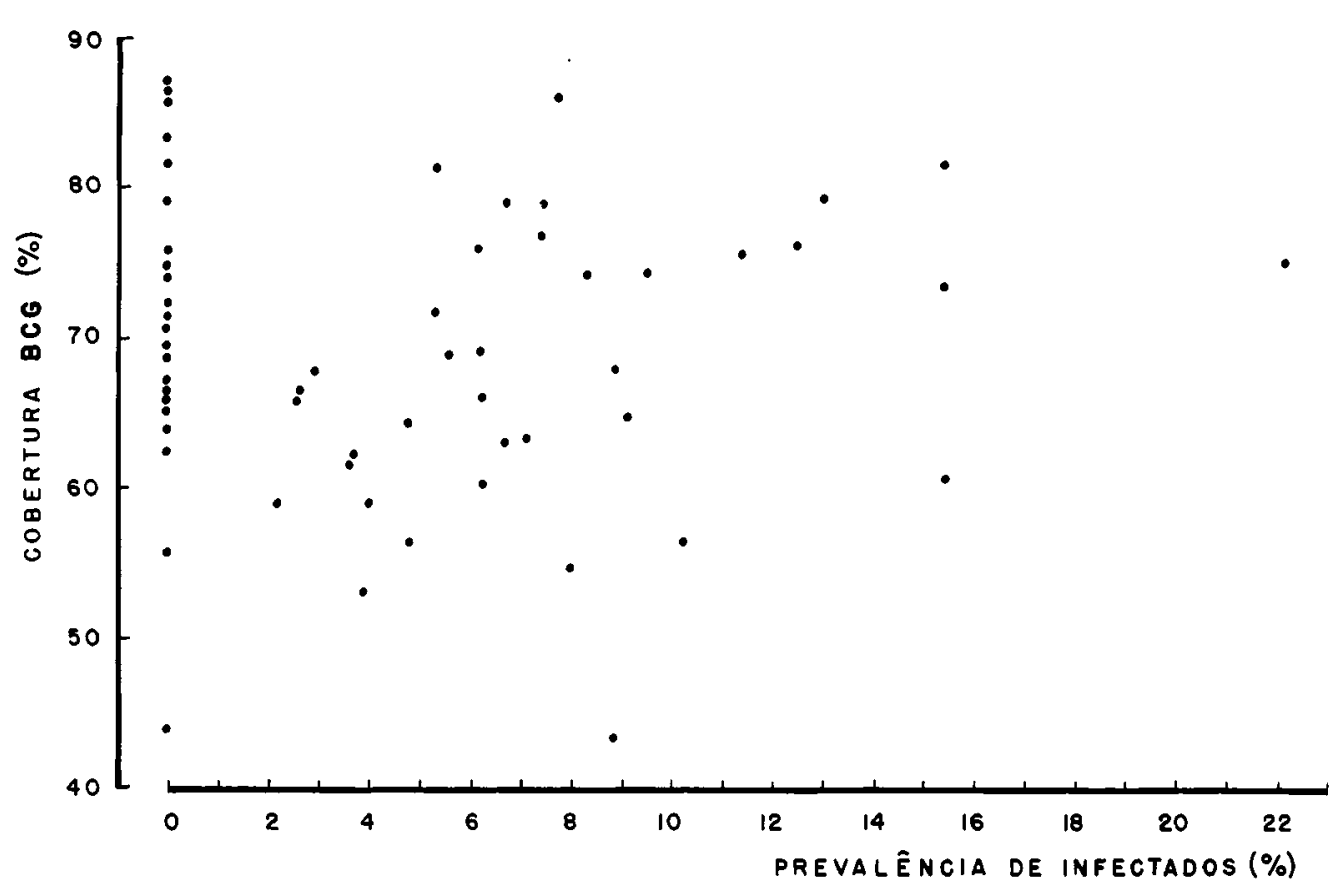

Fig. 5 - Correlação entre a cobertura BCG e d prevalência de infectados aos 7 anos de idade. em 59 escolas municipais de São Paulo, SP, em $1982(r=0,024)$.

\section{DISCUSSÃO}

A metodologia mais adequada para o cálculo do risco anual de infecção e sua tendência, em uma dada população, é aquela que se baseia na prevalência da infecção tuberculosa em diversas idades, em anos diferentes ${ }^{25}$. Os dados disponíveis, relativos ao período de 1970 a 1976, poderiam ter sido utilizados para esse fim, não fosse a falta de comparabilidade entre os mesmos. Assim, os resultados relativos ao ano de 1970 referem-se a alunos de 104 escolas estaduais ( $20 \%$ do total), cuja distribuição geográfica não coincide com as das escolas municipais; de 1971 a 1974 referem-se a alunos de todas as escolas municipais existentes na época (pouco mais de 200); em 1975 o levantamento foi realizado em apenas $10 \%$ das escolas municipais e em 1976 em outra amostra de $10 \%$, diferente da anterior. A sequiência das escolas não seguiu a mesma ordem nos sucessivos levantamentos de modo que uma mesma escola foi pesquisada, nos diversos anos, em diferentes momentos do ano letivo, o que prejudica a comparação. A razão para isso é a evasão escolar, fenômeno ainda significativo no ensino público deste Estado ${ }^{18,21,22}$, inclusive no decurso da primeira série do primeiro grau, sendo lícito supor que os evasores sejam os de pior condição sócio-econômica e por isso mais sujeitos ao risco de infecção tuberculosa. Consequientemente, os dados referentes ao segundo semestre, provavelmente expurgados da parcela mais afetada, não podem ser misturados com os dados relativos ao primeiro semestre. Na Tabela 2 pode-se ver que no ano de 1974 a prevalência de reatores fortes, do primeiro para o segundo semestre caiu de $7,4 \%$ para $5,7 \%$, embora as escolas examinadas no segundo semestre estivessem localizadas nas zonas mais carentes da cidade.

O exame dos dados da Tabela 1 revela que a prevalência por idade foi bas- 
ARANTES, G. R. et. al. Estudo sobre a evolução do risco de infecção tuberculosa em área com elevada cobertura por BCG. Rev. Saúde públ., S. Paulo, 19:95-107, 1985.

tante irregular nos anos de 1973, 1975 e 1976, quando o lógico seriam valores regularmente em ascensão; já a irregularidade da prevalência às idades de 6 , 8 e 9 anos, na sequiência dos anos estudados, seria explicada pelo pequeno número de alunos com essas idades, cursando a primeira série do primeiro grau. O material mais apropriado seria aquele referente à idade de 7 anos, no período de 1971 a 1974 , desde que se pudesse analisar separadamente cada semestre - o que não foi possível devido à perda dos dados primários relativos aos 3 primeiros anos.

Daí o motivo pelo qual, de todo o material anterior, só foi aproveitado o grupo etário de 7 anos, referente ao ano de 1974.

Sendo a cobertura BCG muito elevada desde os primeiros anos de vida, correse o risco de incluir inadvertidamente alguns vacinados sem cicatriz, no grupo dos não vacinados ${ }^{18}$. No material desta pesquisa, 52 crianças vacinadas que não apresentavam cicatriz foram identificadas pelo dado positivo nas cadernetas de vacinação. Acontece que $53 \%$ das crianças sem cicatriz (Fig. 2) não apresentaram aquele documento, impossibilitando a identificação de outros vacinados sem cicatriz, que devem ter feito parte, erroneamente, do grupo não vacinado. Supondo-se que a distribuição fosse a mesma, estimou-se que 60 deles estariam nessa categoria, e com isso o número total de vacinados sem cicatriz andaria por volta de 112 (3\%), o que está dentro da proporção esperada. Tais crianças, efetivamente não identificadas, certamente "contaminaram" o perfil tuberculínico do grupo não vacinado e, em contrapartida, propiciaram uma subestimativa do perfil dos vacinados.

Quando a estratégia de vacinação é a direta, isto é, sem teste tuberculínico prévio (como ocorre em São Paulo), os não vacinados podem ser utilizados em estu- dos de risco de infecção. Nessa eventualidade aceita-se que os mesmos representem tanto os não-reatores como os reatores, anteriores à vacinação ${ }^{16}$. Todavia, a comparação entre os dados de 1982 com os da população estudada em 1974 talvez não seja válida se a cobertura BCG influir na incidência e prevalência de infecção entre os não vacinados, em termos reais ou apenas afetando os cálculos. Nesse sentido, pesquisadores do Instituto Nacional de Tuberculose, da India, fizeram uma investigação para estudar a possibilidade de variações na prevalência de infecção em crianças não vacinadas, em 90 localidades do interior daquele país, em função de diferentes coberturas com BCG; não foi observada correlação entre as duas variáveis ${ }^{9}$. Mais ainda, a prevalência de infecção nos grupos examinados não foi diferente da observada em 119 localidades vizinhas nas quais a vacinação BCG era quase ausente ${ }^{10}$.

Neste trabalho os dados de cobertura vacinal e de prevalência de infecção, nas 59 escolas estudadas, também evidenciaram não correlação entre ambas (Tabela 6 e Fig. 5), e por isso, os resultados de 1974 foram comparados com os de 1982.

A tendência da prevalência de infecção (e, consequientemente, do risco de infecção) revelou-se decrescente no total das escolas examinadas (Tabela 3); analisada segundo as regiões administrativas da Secretaria Municipal de Educação, também foi decrescente, com exceção da regional da Sé. Esse fato pode decorrer dos muitos cortiços e barracos isolados existentes na área, mas o pequeno número de crianças examinadas, em 1982, também poderia explicar a discrepância observada.

Análise segundo o Distrito ou Subdistrito de Paz (Tabela 4) revelou tendência decrescente no sub-conjunto dos mesmos onde mais de uma escola foi 
ARANTES, G. R. et. al. Estudo sobre a evolução do risco de infecção tuberculosa em área com elevada cobertura por BCG. Rev. Saúde públ., S. Paulo, 19:95-107, 1985.

pesquisada (de $7,3 \%$ para $4,4 \%$ ); este grupo compreende $86 \%$ das crianças examinadas. Nos demais distritos/subdistritos, onde apenas uma escola foi pesquisada, a tendência foi variável porém a resultante final foi ascendente de $7,8 \%$ para $9,1 \%$. E possível que o pequeno número de participantes, especialmente no segundo inquérito, explique a variabilidade observada. Não se pode afastar, porém, a possibilidade de um real incremento no risco de infecção em algumas áreas, acompanhando a notória desvalorização do poder aquisitivo e piora da qualidade de vida de uma substancial parcela da população metropolitana.

Estudo realizado em 1975 com os dados da Tabela 1 , sem que se tivessem levado em conta as restrições apresentadas no início deste capítulo, levou à conclusão de que o risco de infecção apresentava tendência ascendente no município de São Paulo, de 1955 a 1971, à base de $6,4 \%$ ao ano ${ }^{15}$. Contraditoriamente, nesse mesmo estudo, a incidência de meningite tuberculosa em crianças menores de 5 anos, a qual costuma manter estreita relação com o risco de infecção, caiu $6,6 \%$ ao ano, no mesmo período! Se esta última for aceita como a expressão mais correta da tendência do risco anual de infecção tuberculosa no período em questão, verifica-se que o declínio observado de 1974 a 1982 foi menor que o declínio de 1955 a 1971 , corroborando a idéia de que a situação epidemiológica da tuberculose estaria piorando na Capital de São Paulo, pelo menos em algumas de suas áreas. $\mathrm{Na}$ falta de dados intermediários entre os dois pontos extremos da curva não se pode afastar a possibilidade de uma tendência em curva parabólica, na qual o risco de infecção, embora com uma resultante final decrescente, esteja aumentando nos últimos anos do período.

Se a tendência do risco de infecção, apesar das falhas apontadas, pôde ser aproximadamente estabelecida, o mesmo não se poderá dizer quanto à sua magnitude nos dois momentos estudados. Nesse sentido, o material de 1974 é incompleto, pois os resultados relativos ao segundo semestre deixaram de incluir evasores provavelmente infectados; e os dados do primeiro semestre não incluíram escolas das zonas leste, sul e noroeste, sabidamente sob condições de vida inferiores às das demais zonas.

Pelos mesmos motivos, o risco referente ao ano de 1982 também pode estar subestimado. Acrescente-se que, cerca de $25 \%$ das crianças de 7 anos estão fora da escola no município de São Paulo 22 .

Finalmente, no que concerne à evolução da alergia tuberculínica no grupo vacinado, os resultados apresentados na Tabela 5 mostram que a mesma ainda. é intensa aos 7 anos de idade, independentemente dos anos transcorridos; os perfís tuberculínicos apresentados nas Figs. 3 e 4 são bastante diferentes entre si, o primeiro deles lembrando a metade direita de uma distribuição normal, como se fosse um perfil em involução, e o segundo, um perfil com tendência à bimodalidade, típico de populações não vacinadas vivendo em áreas com baixa prevalência de micobactérias atípicas ${ }^{3}$.

\section{CONCLUSOEES}

À vista dessas considerações os autores chegam às seguintes conclusões:

1. A resultante final da evolução do risco de infecção tuberculosa, de 1974 a 1982, na população estuda$\mathrm{da}$, foi decrescente, embora não se possa afastar uma componente ascendente nos últimos anos do período.

2. Novos estudos sobre o assunto deverão levar em conta a evasão escolar, incluir a rede pública de ensino estadual e a rede privada e abranger todos os Distritos/Sub-distritos da cidade. 
ARANTES, G. R. et. al. Estudo sobre a evolução do risco de infecção tuberculosa em área com elevada cobertura por BCG. Rev. Saúde públ., S. Paulo, 19:95-107, 1985.

3. Nas escolas estudadas não houve correlação entre a cobertura vacinal e a prevalência de infecção natural aos 7 anos de idade.

4. A alergia tuberculínica no grupo de crianças com 7 anos completos, va- cinadas em alguma idade entre $o$ primeiro e o sexto ano de vida, revelou-se 2,5 vezes mais intensa que a alergia em um grupo de crianças da mesma idade, não vacinadas, o que inviabiliza a sua utilização para o cálculo do risco de infecção.

ARANTES, G, $R$, et al. [The trend in the risk of tuberculous infection in an area with wide coverage with BCG vaccination]. Rev. Saúde públ., S. Paulo, 19:95-107, 1985

ABSTRACT: The estimation of the risk of tuberculous infection from prevalence data obtained at school-age, in 1974 and in 1982, permitted the determination of the relevant trend in the city of S. Paulo, Brazil, between those years. The risk of infection decreased, on average, by $5 \%$ annually during the period. There was no evidence of any association between the proportions of vaccinated children and that of infected children among those unvaccinated, in the 59 schools studied. Tuberculin sensitivity in 7 years old school-children, yaccinated with BCG at any age between the 1 st and the $6^{\text {th }}$ year of life was 2.5 times more intense than that in unvaccinaetd children of the same age. With regard to the calculation of the true value of the risk of tuberculous infection, commentaries about the unrealiability of the available data were made.

UNITERMS: Tuberculosis BCG vaccine. Tuberculin test. Hipersensibility, delayed.

\section{REFERENCIAS BIBLIOGRÃFICAS}

1. ALMEIDA, E.S. et al. Infecção tuberculosa natural e o uso de BCG oral e intradérmico em escolares de Laranjal Paulista, SP, Brasil. Rev. Saúde públ., S. Paulo, 7:189-97, 1973.

2. ALMEIDA, E.S. et al. Nível de infecção tuberculosa e viragem tuberculínica pelo BCG intradérmico em escolares de Botucatu (SP) Brasil, em 1969. Rev. Saúde públ., S. Paulo, 8:31-41, 1974.

3. ARANTES, G.R. et al. Interpretação da sensibilidade tuberculínica em popula. ção do interior do Estado de São Paulo. Rev, Saúde públ., S. Paulo, 10:219. 26, 1976.

4. BLEIKER, M.A. Epidemiological trends in low prevalence countries. Bull. int. Un. Tuberc., 49:128-35, 1974.

5. BROLIO, R, et al. Risco de infecção tuberculosa no primeiro ano de vida, n'uma área do município de São Pau. lo, 1970-74. Rev. Div. nac. Tuberc., 20: $391-5,1976$.

6. CARANDINA, L. Infecção tuberculosa em escolares da zona urbana de Botucatu, São Paulo, 1976. São Paulo,
1980. [Dissertação de mestrado - Faculdade de Saúde Pública da USP].

7. CERTAIN, D.A, et al. Levantamento da prevalência da infecção tuberculosa em escolares do primeiro ano primário das escolas públicas de São Paulo, 1970. Rev. Saúde públ., S. Paulo, 6:189-97, 1972.

8. CERTAIN, D.A, et al. Análise dos resultados da pesquisa da infecção tuberculosa e do primeiro programa de vacinação pelo $\mathrm{BCG}$ intradérmico em escolares de São Paulo, Brasil, 19711974. Rev. Saúde públ., S. Paulo, 9: 125-36, 1975.

9. CHARKRABORTY, A.K, et al. Prevalence of infection among unvaccinated children for tuberculosis surveillance. Indian J. med. Res., 72:7-12, 1980.

10. GOTHI, G.D. et al. Prevalence of tuberculosis in a South Indian district. Indian J. Tuberc., 26:121-35, 1979.

11. HARRIS, W.M. Importância sanitária do cadastro computadorizado escolar. São Paulo, 1977. [Dissertação de mestrado - Faculdade de Saúde Pública da USP] 
ARANTES, G. R. et. al. Estudo sobre a evolução do risco de infecção tuberculosa em área com elevada cobertura por BCG. Rev. Saúde públ., S. Paulo, 19:95-107, 1985.

12. LESER, W. \& BARBOSA, V. Relacionamento de certas características populacionais com a mortalidade infantil no município de São Paulo, de 1950 a 1970. Probl. bras., 10:17-30, 1972.

13. MAGALDI, C. et al. Aspectos epidemiológicos da tuberculose pulmonar nos municípios de Botucatu, Conchas, São Manuel e Avaré, Estado de S. Paulo, Brasil, de 1963 a 1972. Rev. Saúde públ., S. Paulo, 10:291-314, 1976.

14. MONTEIRO, C.A. et al. Mortalidade no primeiro ano de vida e a distribuição de renda e de recursos públicos de saúde, São Paulo (Brasil). Rev. Saúde públ., S. Paulo, 14:515-39, 1980.

15. MORAES, J.C. de Risco de infeç̧ão tuberculosa e incidência de meningite tuberculosa. São Paulo, 1977. [Dissertação de mestrado - Faculdade de Medicina da USP]

16. ORGANIZACIÓN PANAMERICANA DE LA SALUD. Control de tuberculosis en America Latina: manual de normas y procedimientos para programas integrados. Washington, D.C., 1979. (Publ. Cient,, 376).

17. PROVA Tuberculínica em Saúde Pública (2. ${ }^{a}$ recomendação). Rev. Serv. nac. Tuberc. 12:219-30, 1968.

18. PRUKS, L.R. Algumas considerações sobre fatores de evasão. São Paulo, Secretaria de Estado da Educação, 1982. [Mimeografado]

19. RUFFINO NETTO, A. \& ARANTES, G.R. Risco de infecção tuberculosa em município do interior do Estado de São Paulo e suas aplicações. Rev. Saúde públ., S. Paulo, 10:143-9, 1976.
20. SECRETARIA DE ESTADO DA EDU$\mathrm{CAÇÃO}$. Rede estadual de ensino: es. tabelecimentos de $1 .^{\circ}$ e $2 .^{\circ}$ grau região metropolitana da Grande São Paulo. São Paulo, 1982. (Série documentos).

21. SECRETARIA DE ESTADO DA EDUCAÇÃO. Educação e desenvolvimento social. Sub projeto I: estudo da implantação da escola de 8 anos. São Paulo, 1983.

22. SECRETARIA DE ESTADO DA EDUCAÇÃO. A permanência do aluno na escola: rendimento escolar no Estado de São Paulo. São Paulo, 1983. [Mimeografado]

23. STYBLO, K. Epidemiologia de la tuberculosis. Bol. Un. int. Tuberc, 53:14557, 1978.

24. STYBLO, K. \& SUTHERLAND, I. Epidemiological indices for planning, surveillance and evaluation of tuberculosis programmes. Bull. int. Un. Tuberc., 49:66-73, 1974.

25. SUTHERLAND, I. Recent studies in the epidemiology of tuberculosis, based on the risk of being infected with tubercle bacilli. $A d v$. Tuberc. Res., 19: $1.63,1976$

26. TORNELL, E. The infection risk for tuberculosis in an industrial city, together with an examination as to the durability of the tuberculin positiveness. Acta tuberc. Scand., 17:261-81, 1943.

Recebido para publicação em 05/09/1984

Reapresentado em 29/01/1985

A provado para publicação em 27/02/1985 